European Association for the Development of Renewable Energies, Environment and Power Quality
International Conference on Renewable Energies and Power Quality (ICREPQ’09)

Valencia (Spain), 15th to 17th April, 2009

\title{
Fuel consumption minimization of a cogeneration system multi machines associated with a photovoltaic
}

\author{
Mélissa Dondas*(1), Firas Alkhalil*(1), Philippe Degobert*(1) , Frédéric Colas*(1) and Benoit Robyns*(2) \\ * Laboratoire d'Electrotechnique et d'Electronique de Puissance de Lille (L2EP). \\ (1) Arts et Métiers ParisTech, L2EP, Lille, France \\ Phone/Fax number: +33 (0)3-20-62-22-29/+33(0)3-20-62-27-50 \\ melido5@hotmail.com firas.alkhalil-8@etudiants.ensam.fr philippe.degobert@ensam.eu frederic.colas@ensam.eu \\ (2) Ecole des Hautes Etudes d'Ingénieurs, L2EP, Lille, France \\ Phone/Fax number: +33 (0)3-28-38-48-58/ +33 (0)3-28-38-48-04 \\ benoit.robyns@hei.fr
}

\begin{abstract}
The minimization of the production costs and the consumption of the electricity are major stakes in the company strategy led by the specialists of the energy sector. This paper describes the research works on the supervision of a hybrid power plant. This project consisted in conceiving and in overseeing a hybrid multi sources system with Distributed Generators associating an existing photovoltaic power plant with a power plant of small multi-cogeneration hatch in order to, first, mitigate the irregularity of the photovoltaic sources, and in a second time, to participate in the ancillary service during the daily points thanks to a management and an appropriate supervision. The main objective is the optimization of the cogeneration system fuel consumption and this work recovered from Unit Commitment Problem that is to define at a lower cost the organization of starting / extinction of every power plant as well as its production over all the period considered so as to satisfy all the constraints. These constraints, besides the satisfaction of the demand of the load, correspond to the respect for the dynamics of every sub-system.
\end{abstract}

\section{Keywords}

Supervision, cogeneration, gas turbine, photovoltaic, hybrid central, energy, minimization, fuzzy logic.

\section{Symbols and notations}

$T$ belonging to $\mathrm{D}=\left(0, \ldots, \mathrm{t}_{\max }\right)$, represents a date $U$ belonging to $U=\left(1, \ldots, \mathrm{u}_{\max }\right)$ that identifies $\mathrm{a}$ production unit.

$d(t)$ : energy demand to satisfy for any date $t \mathrm{D}+$. $d_{\max }(t)$ : maximum demand for energy to be guaranteed, taking into account the power stations lit at the date $\mathrm{T}$ (taking into account of $10 \%$ of reserve).

$y_{u}(t) \in\{0,1\}$ : operation indicator of the unit $\mathrm{u}$ at the time $\mathrm{t}$. $y=1$ if unit $\mathrm{u}$ is active at the time $\mathrm{t}$ and 0 otherwise.

$s_{u}(t)$ : belonging to $\mathrm{Z}^{+}$: variable state of the unit $\mathrm{u}$ at the time $\mathrm{t}$ associated with the command $\mathrm{y}_{\mathrm{u}}(\mathrm{t})$.

$p_{\text {min } ~}$ : Provided minimum power

$p_{\max u}$ : Provided maximum power

$t_{\text {up } ~}$ : Minimum duration of starting.

$t_{\text {down } u}:$ Minimum duration of extinction

$c_{h s u}:$ Cost Hot Start.

$c_{c s u}:$ Cost Cold Start.

$t_{c s u}$ : Time Cold Start.

$c_{f u}(p)=a_{0 u}+a_{1 u} \cdot p+a_{2 u} \cdot p^{2}:$ The fuel cost function of each unit is taken as a quadratic function.

$p_{u}(t)$ belonging to $\mathrm{R}^{+}$: Power produced by the unit at time $\mathrm{T}$.

\section{Introduction}

Unit commitment problem (UCP) [5-7] and [3] is the problem of selecting the generating units to be in service during a scheduling period and for how long. The overall problem can be divided into two sub problems namely unit commitment and economic dispatch. The committed units must meet the system load and reserve requirements at minimum operating cost, subject to a variety of constraints. The consumption minimization of generators is a topic that has been the subject of numerous studies and scientific publications, especially as regards the UCP. Different approaches of resolution exist even if the formulation of the problem remains unchanged from one article to another.

Microgrids [9, 12] are low-voltage distribution systems that combine, electrical distributed sources (microturbines, fuel 
cells and photovoltaic), energy storage devices (batteries, capacitors) and controllable loads. These energy systems can, through the use of renewable energy and other micro-sources, reduce emissions of greenhouse gases while maximizing the reliability of electricity supply.

The mathematical formulation of this problem (UCP) is a non-linear optimization problem mixed with discrete and continuous variables, which combines a non-linear objective function and coupled constraints. The optimal solution of this complex optimization problem for energy production processes can be obtained by techniques for global research [1], but they have the disadvantage of computing time and memory which used too much. The Lagrangian relaxation [2] is more effective to this point of view and it has interest to the problems of large scale. But the problems of convergence can provide. Other methods, using genetic algorithms, fuzzy logic or research taboo [4] - [8], show accurate results in interesting times.

A strategy, using fuzzy logic, is proposed [12] to participate in the ancillary services by adjusting the frequency and voltage. Finally, the present paper proposes a methodology for assessing and minimizing the fuel consumption costs and electricity production costs.

The plant studied shown in Fig.1 consists of a photovoltaic power of a peak about $20 \mathrm{kWe}$, of a two gas generators $(30 \mathrm{~kW}, 25 \mathrm{~kW})$ and of a storage capacity $(16.5 \mathrm{~F} / 480 \mathrm{~V},-66 \mathrm{kWe}$ à $66 \mathrm{kWe})$. The charge was modeled by a daily load profile of an airport in the region. In the same way, sunshine daily profiles recorded on the "Arts et Métiers ParisTech" facilities were used to simulate the production of renewable energy.

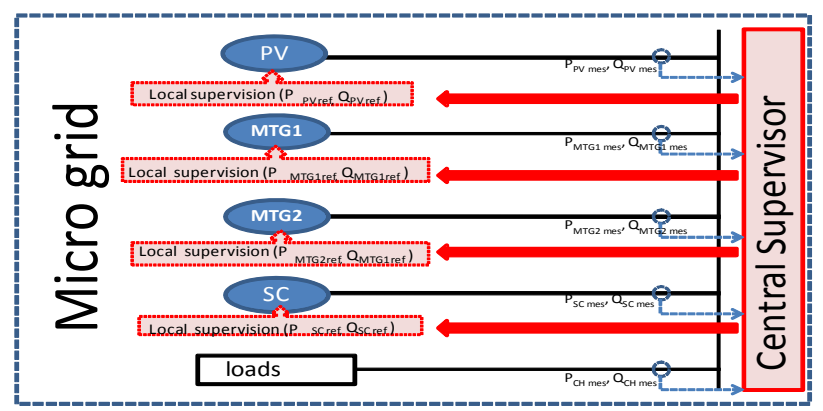

Fig.1. Plant basic elements

\section{Unit Commitment Problem}

The goal [3, 6, 7] of unit commitment problem is to decide which of the available generators should start up and shut down over a given time horizon so that the overall operating cost is minimised subject to demand and spinning reserve constraints.

\section{A. Objective function:}

The UCP objective function [3, 2] is expressed as the sum of fuel cost, the start up and shut down cost of individual units for the given period subjected to various constraints. Mathematically it can be formulated as follows:

$$
(P):\left\{\begin{array}{l}
\min _{p, y} f(p, y)=\sum_{u \in U} \sum_{t \in D^{+}} c_{\mathcal{P}_{u}}(t)=\sum_{u \in U} \sum_{t \in D^{+}}(\underbrace{y_{u}(t) \cdot c_{u}\left(p_{u}(t)\right)}_{\text {operation }}+\underbrace{c_{u s}\left(t, y_{u}\right)}_{\text {starting }}) \\
\text { under the oonstraints } \quad \text { C1 à C5 }
\end{array}\right.
$$

The operation cost of a unit $u$ is expressed as a non-linear function of the produced power $\mathrm{p}_{\mathrm{u}}$ :

$$
c_{f u}\left(p_{u}\right)=a_{0 u}+a_{1 u} \cdot p_{u}+a_{2 u} \cdot p_{u}^{2} .
$$

The start up cost of the unit is taken into account only once every start up. Its value depends on the state of the cooling unit, knowing a unit cold start more expensive to a unit hot start.

$$
c_{\mathrm{s} u}(t)=\left\{\begin{array}{lll}
c_{\mathrm{hs} u} & \text { si } & t_{\mathrm{off} u}<t_{\mathrm{cs} u} \\
c_{\mathrm{cs} u} & \text { si } & t_{\mathrm{off} u} \geq t_{\mathrm{cs} u}
\end{array}\right.
$$

Here, $t_{\text {off } u}$ belonging to $\mathrm{N}$ : duration of extinction of plant.

$t_{c s} u$ belonging to $\mathrm{N}$ : duration of extinction beyond which the production unit $\mathrm{u}$ is considered cold.

\section{B. Constraints:}

Depending on the nature of the power system under study, The UCP has many constraints such as the power balance, spinning reserve and the other constraints including the thermal constraints, fuel constraints and security constraints.

1) The first constraint is the satisfaction of global need. For each date, the sum of all power plants must equal the demand for this date:

C1 Demand to satisfy $\quad: \forall t \in D^{+}, \quad \sum_{u \in U} y_{u}(t) \cdot p_{u}(t)=d(t)$

2) Managing uncertainty is maintaining a reserve of $10 \%$ over demand

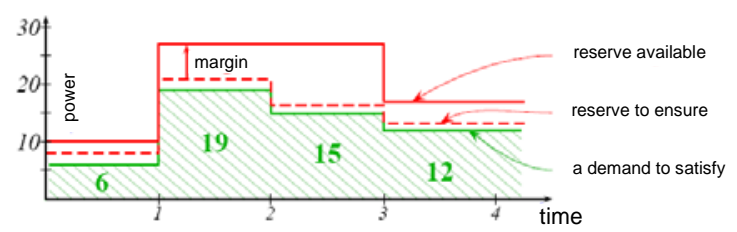

Fig.2. Constraints C1 and C2 [2]

C2 Reserve to ensure : $\quad \forall t \in D^{+}, \quad \sum_{u \in U} y_{u}(t) \cdot p_{\max u} \geq d_{\max }(t)$

Then we express the another technical constraints in the constitution of generating units as followed;

3) First, the production capacity of a unit is limited, between a minimum, below which its performance is diminished (performance degrades) and maximum power.

C3 Power limits : $\quad \forall t \in D^{+}, \forall u \in U, \quad y_{u}(t) \cdot p_{\min u} \leq p_{u}(t) \leq y_{u}(t) \cdot p_{\max u}$ 
$4,5)$ the generators start up orders are subjected to dynamic constraints shown in fig.3, which imply times of inertia on the starting or the extinction of the generators.

C4 Minitime $t_{\text {down } u}: \quad \forall t \in D^{+}, \forall u \in U, \quad \overline{y_{u}}(t-1) \cdot y_{u}(t)=1 \Rightarrow-s_{u}(t-1) \geq t_{\text {down } u}$

C5 Mini time $\quad t_{\mathrm{up}_{u}}: \quad \forall t \in D^{+}, \forall u \in U, \quad y_{u}(t-1) \cdot \overline{y_{u}}(t)=1 \Rightarrow s_{u}(t-1) \geq t_{\mathrm{up}_{u}}$

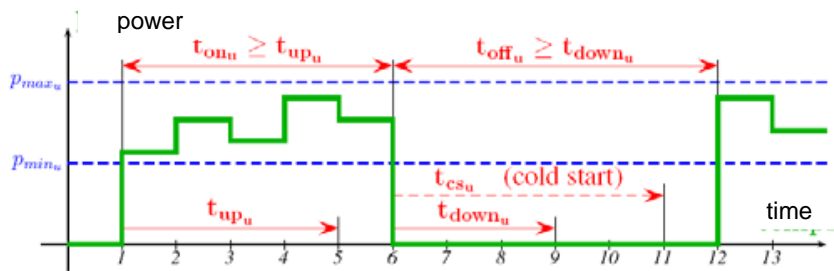

Fig.3: Generators start up orders constraints [2]

\section{Supervision of the hybrid plant}

The fig.4 below shows the microgrid simulation schematic diagram. The supervisor, whose main function is to calculate and send Powers reference to system, consists of two parts: The optimization supervisor and the supervisor based on fuzzy logic.

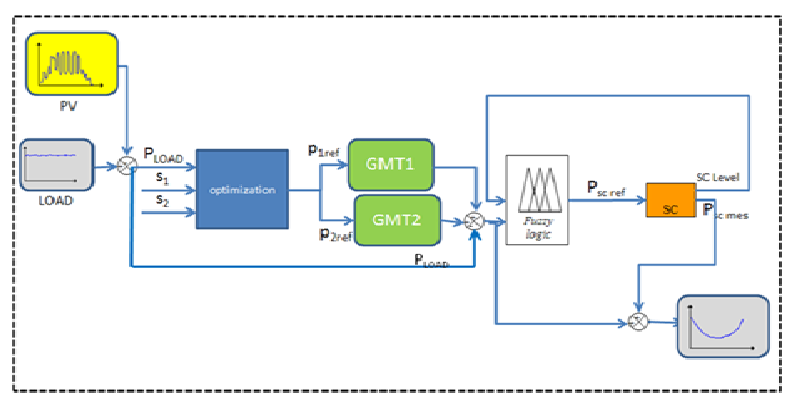

Fig.4: Microgrid simulation schematic diagram

\section{A. Creation of the gas microturbine signals}

As already explained, the generators are subjected to the constraints that prevent immediate restart up a unit after its extinction, or inversely extinction after start up, for reasons of reliability. Minimum durations must be respected before any change in the status of a GMT. To calculate consumption costs, the status of the GMT is paramount. Indeed, the status of the GMT has an immediate impact as a restart hot consumes less fuel than a cold start.

The variable $s_{u}$ already introduced, represents the time from which the unit is turned on (if $\mathrm{s}_{\mathrm{u}}(\mathrm{t})<0$ ) or off (if $\mathrm{s}_{\mathrm{u}}$

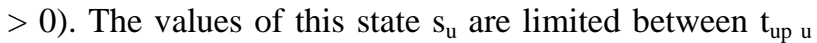
and $t_{c s}$, time beyond which the state of the production unit may be changed without danger. The state of the on / off unit $\mathrm{u}$ is represented by the binary variable $\mathrm{y}_{\mathrm{u}}$. The synthesis of this signal is done by measuring the level of power output of the unit. It is (1) between $\mathrm{p}_{\min u}$ and $\mathrm{p}_{\max } \mathrm{u}$ and (0) below $p_{\min }$ u. Fig.5 below illustrates the evolution of these signals on a day to a production unit.
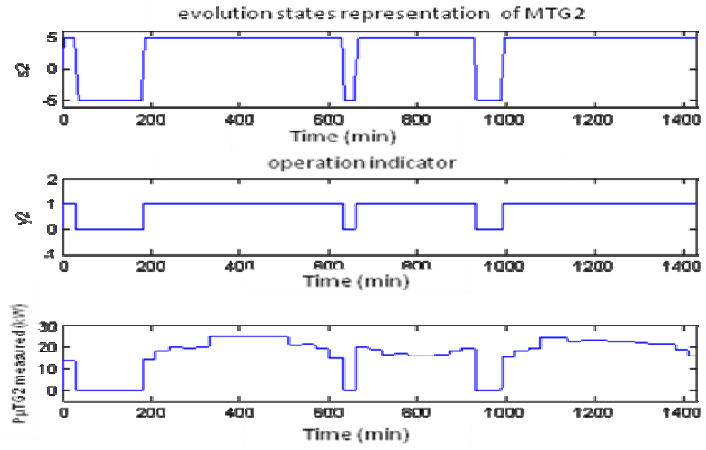

Fig. 5: Evolution of states signals, running and power production unit 2

\section{B. Optimization Algorithm [4, 5]}

The problem resolution can be achieved by using optimization tools that are found in the optimization toolbox of MATLAB Simulink. The constraints and the function to minimize, being defined, the mixed integer optimization algorithms are suitable. The optimization toolbox YALMIP, Internet free access and compatible with MATLAB Simulink, was used for the optimization calculations. The solvemoment function solves the minimization problems defined in part1-A

\section{Supervision by fuzzy logic of reference power of storage element}

Fuzzy logic $[8,17]$ is a powerful method of problem solving that has many applications in embarked control and in information management. It allows to draw conclusions from information vague, ambiguous or unclear. In a sense, fuzzy logic mimics the human decision-making with his ability to find specific solutions from approximate data.

The Fuzzy logic supervisor determines reference power to send to the supercapacitor from its supercapacity energy level (SC Level) and the power data non satisfied by GMT $\left(\mathrm{P}_{\text {fuzzy }}=\right.$ $\mathrm{P}_{\text {LOAD }}-\mathrm{P}_{\mathrm{GMT1}}$ - $\left.^{-} \mathrm{P}_{\mathrm{GMT} 2}\right)$. The following fig. 6 shows the evolution of the supercapacitor reference power $\mathrm{P}_{\mathrm{sc}}$ ref according to SC Level and $\mathrm{P}_{\text {fuzzy. }}$.

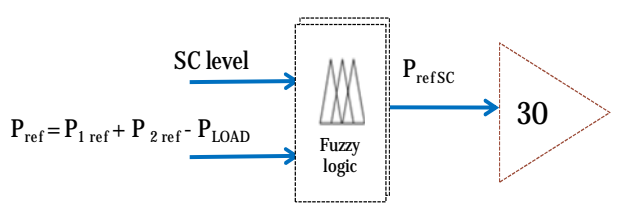

Fig. 6: Evolution observer of PSC ref

\section{Results Analysis}

The cogeneration plant is not permanently solicited to identical powers. Indeed, because of the photovoltaic power, the weather influences significantly the hybrid station overall operation. 
The simulations realized were able to identify different situations depending on weather conditions. In what follows, we will consider two scenarios:

1. Case the most favorable: cold and sunny day

2. Unfavorable case: very cloudy day

\section{A. Scenario1: cold and sunny day}

Fig.7 illustrates the daily load profile of an airport and the production of photovoltaic power on 24. The power of reference which will be forwarded to the cogeneration station is the difference between the load and power generated by solar panels. As you can see the waveform of the power generated by the station PV is quite regular. It corresponds to weather a cold and sunny day, i.e that there is no fast and irregular power change. Fluctuations occur in a progressive manner depending on the angle of sunlight compared with photovoltaic panels, with a maximum power which is around $13 \mathrm{~kW}$.

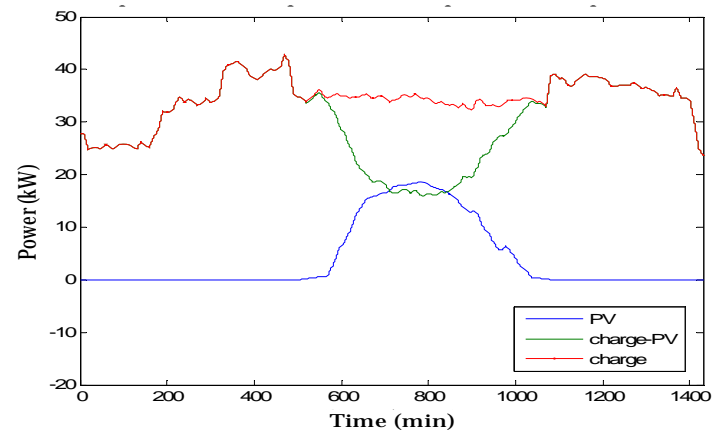

Fig.7: Load profile and the production of photovoltaic power on $24 \mathrm{~h}$

The reference power represented in green, will be sent to optimization calculator . The calculator will, through an optimization algorithm that minimizes costs, distribute the power between the different GMT.

we see on fig.8 and fig.9, the distribution on two GMT of the power demand for optimization cadency worth respectively 5 and $120 \mathrm{~min}$. The optimization cadency represents the period after which the algorithm calculates the reference power of each machine according to the present power demand. The optimization frequency has a significant impact on the final cost as we can thereafter see . On these two figures, we can find the same time scale where GMT are solicited or not.

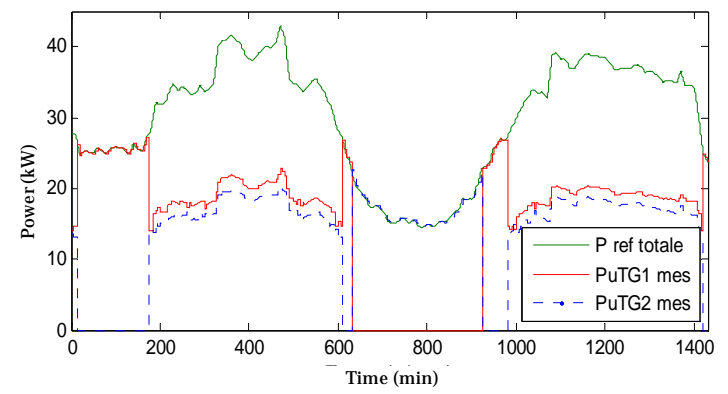

Fig.8: Produced power by both GMT, optimization cadency $=5 \mathrm{~min}$

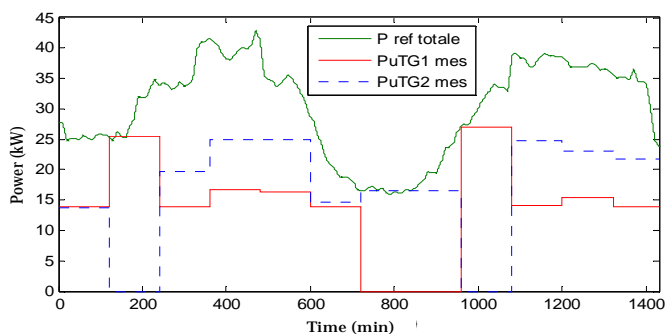

Fig.9: Produced power by both GMT, optimization cadency $=120 \mathrm{~min}$

For optimization cadency of 5 minutes, the total produced power by the two microturbines (see Fig.10) follows perfectly fluctuations of the load, which is not the case for a cadency of 120 minutes. In the latter case, the use of supercapacitor is needed to store power surplus or to fill in the lack of energy, since there are significant differences between the reference and power production GMT (dashed red curves and solid dark green).

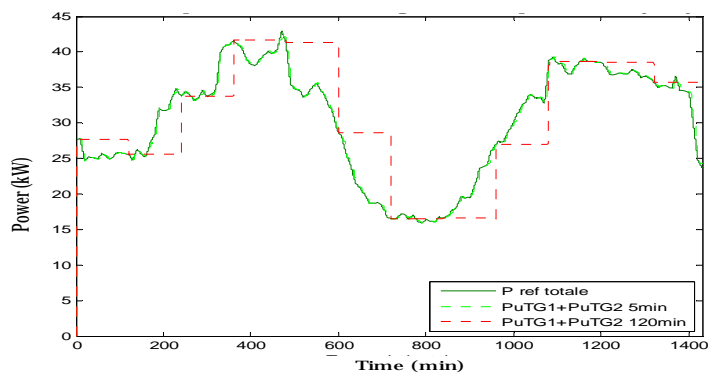

Fig.10: Total production of the cogeneration plant, sunny day

The function of supercapacitor is also to fill in the lack of power caused by slow dynamics of GMT

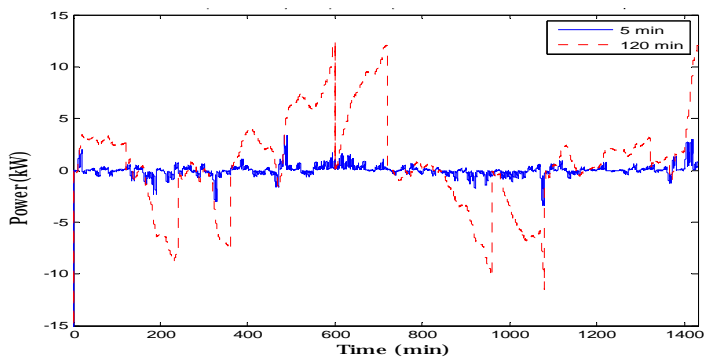

Fig.11: Provided power by the supercapacitor for different optimization cadencies.

We note on the fig.11, the presence of peak power of amplitude lower (up to $3.5 \mathrm{~kW}$ maximum) for optimizing every 5 minutes than for an optimization performed every 2 hours (maximum amplitude of $13 \mathrm{~kW}$ ). The capacities are in a case where voltage variations more frequent but smaller amplitude. The frequency and amplitude of charge and discharge will obviously affect the life of supercapacitor. The curves of Fig.12 represent the fuel consumption at a given time of the cogeneration plant to the different optimization cadency. They have the same apparence (air) [15] than the curves of cogeneration power. 


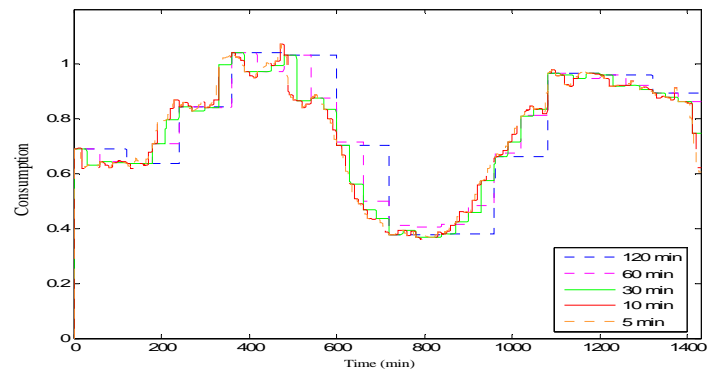

Fig.12: Consumption evolution of GMT depending on the optimization cadency for a sunny day

Fig.13 illustrates the cost evolution for different optimization cadencies . The total cost is the cumulative consumption costs at the end of the day. we find that it is much greater when the optimization cadency is less than 30 minutes (cadency every 5 or $10 \mathrm{~min}$ ). The references powers of GMT follow to the power fluctuations charge nearest for these cadencies.

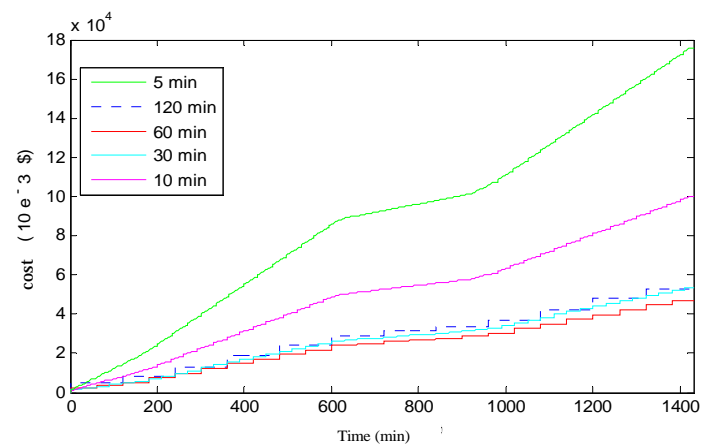

Fig.13: Consumption cost of GMT for a sunny day

There is a greater cost to a calculation every 2 hours, slightly superior to the cost of optimization every half hour or every hour.

\section{B. Scenario2: Cloudy day}

At a cloudy day, the production of PV power is subject to fluctuations, which can be slow or fast as shown in Figure 14 below.

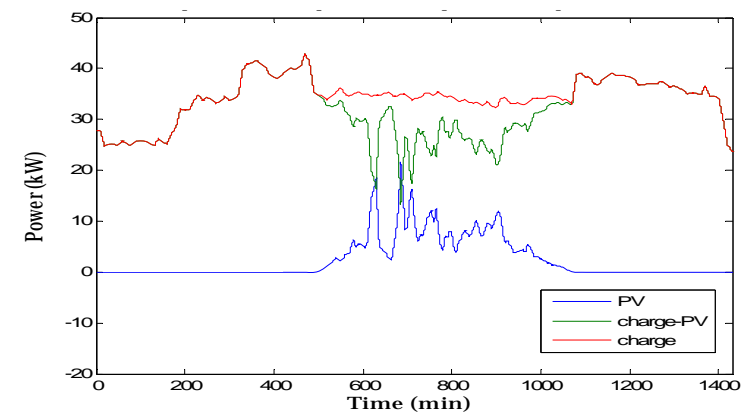

Fig.14: Load profile and the production of photovoltaic power on $24 \mathrm{~h}$

the references Powers of GMT will also undergo rapid changes as shown in Fig.15 and fig.16 showing the repartition of power between the two microturbines.

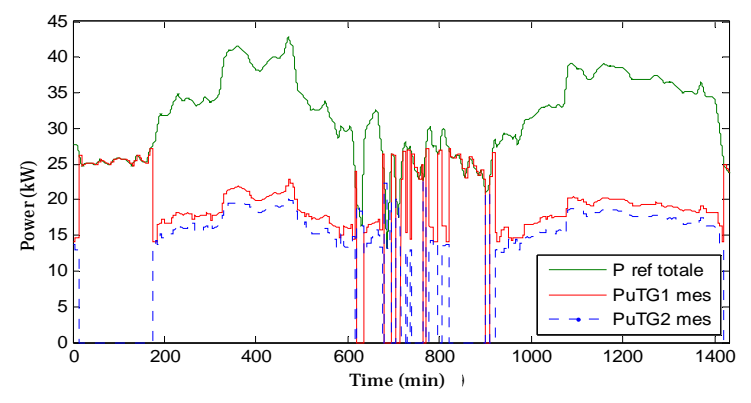

Fig.15. Power produced both GMT, optimization cadency $=5$ $\min$

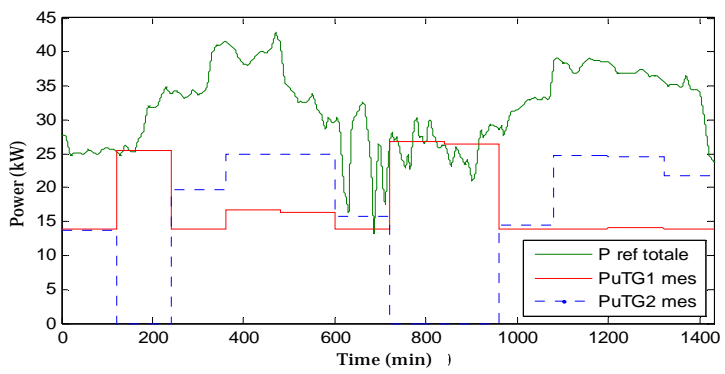

Fig.16: Power produced both GMT, optimization cadency $=120 \mathrm{~min}$

In fig. 15 above, we notice a zone between 600 minutes and 900 minutes, where GMTs are solicited due to the number of starts and stops in this zone. Indeed, it accelerates the gas turbine aging and generates a cost not immediately postponed because over time if there is a change in the cogeneration equipment. With regard to the optimization every two hours, we see the figure 16 that we get pretty much the same number of stop / start for a sunny day.

Fig.17 following shows the changes in power at the terminals of the supercapacitor. For fast optimization of 5 minutes (in blue), changes in power are rapidly maked, with such a power of $11 \mathrm{~kW}$ provided in 4.5 minutes.

This means that the physical supercapacitor will be able to provide such powers in a considered time. But in the same way that fluctuations too running references of GMT are harmful, the frequent changes too and too high amplitude decreases the life of supercapacitor.

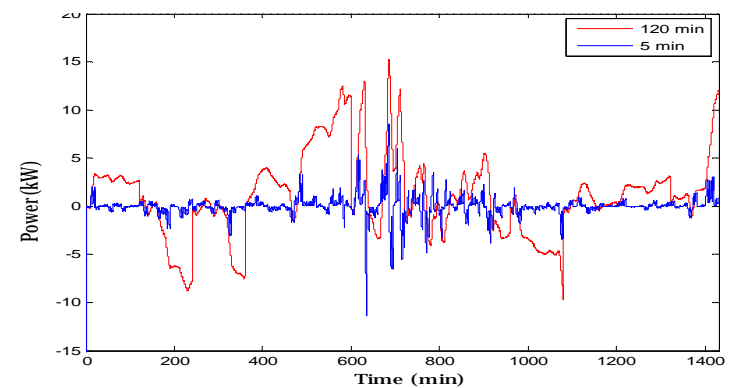

Fig.17: Provided power by the supercapacitor for different optimization cadencies

Fig.18 shows the total consumption in real time for two microturbines. It is always proportional to the production of microturbines as in the previous case. 
In fig.18, we notice growth change exponentially consumption costs of GMT for cadencies below $30 \mathrm{~min}$, with higher values in the case of a cloudy day than a sunny day.

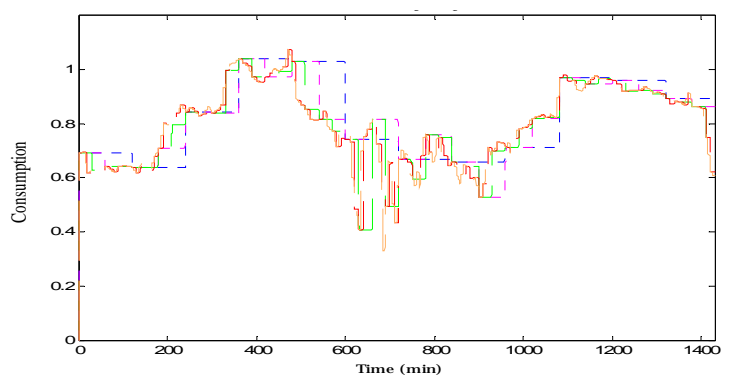

Fig.18: Consumption evolution of GMT depending on the optimization cadency for a cloudy day

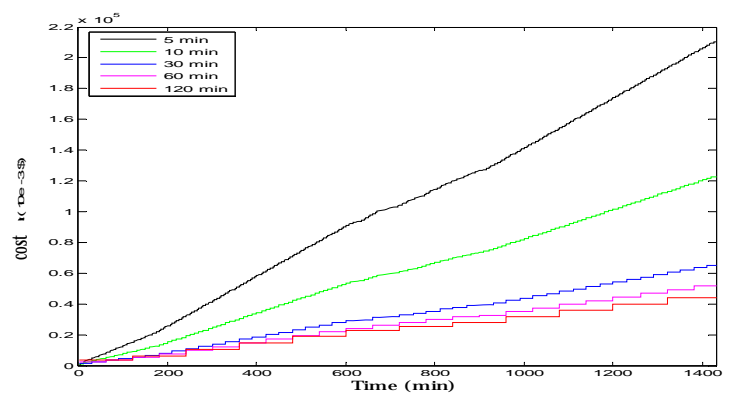

Fig.19: Consumption cost of GMT for a cloudy day

\section{Conclusion}

The Supervision of hybrid Central minimizing the fuel consumption of the cogeneration device has been achieved in this work. first, it was necessary to create the necessary signals for the execution of algorithm. A simple application of fuzzy logic has been implemented to supplement the lack of energy microturbines by supercapacitors. The results highlighted the potential impact of the choice of optimization cadency on the cost evolutions.

A high cadency increases significantly the fuel consumption of microturbines, thereby increasing the overall cost of the plant. It also has the disadvantage of accelerating the aging of these microturbines, because of inopportune stops and starts. However, a reduced optimization cadency has the advantage of a lower consumption of microturbines. But in this case, the supercapacitors are much solicited, and it will take into account the cost of they usury in the Costing.

For the remainder of this project should find a compromise that allow to handle carefully the two devices (microturbines and supercapacitors) realizing an optimizer to a variable cadency for example.

\section{References}

[1]LOWERY P.G., Generation Unit Commitment by Dynamic Programming, IEEE Transaction On Power Systems Vol.102, No.3, 1983, pp.1218-1225.
[2]BENOIST T., DIAMANTINI M., ROTTEMBOURG B., Relaxation lagrangienne et filtrage par coûts réduits appliqués à la production d'électricité. Rapport, Rapport ENSTA, 2005, 30p.

[3]CHANDRAM K., SUBRAHMANYAM N., SYDULU M., Unit Commitment by Secant method combined with IPPD table. IEEE Transaction On Power System, National Institute of Technology, India, 7p.

[4]LIAO G., Application Meta-Heuristics Method for ShortTerm Unit Commitment Problem, IEEE Transaction On Power System, Taiwan, 2004, 5p.

[5]SENJYU T., YAMASHIRO H., SHIMABUKURO K., Fast solution technique for large-scale unit commitment problem using genetic algorithm, IEE Proceedings Generation, Transmission and Distribution, Japan, November 2003, pp.753-760

[6]RAJAN C., MOHAN M., MANIVANNAN K., Neuralbased tabu search method for solving unit commitment problem, IEE Proceedings - Generation, Transmission and Distribution, India, July 2003, pp.469-474

[7]MORI H., MATSUZAKI O., A Parallel Tabu Search Approach to Unit Commitment in Power Systems, IEEE Transaction On Power System, Japan, 1999, pp.509-514

[8]LIAO G., TSAO T., The use of genetic algorithm/fuzzy system and tabu search for short-term unit commitment, IEEE Transaction On Power System, Taiwan, 2002, pp.2302-2307

[9]DENDA A., Shimizu's Microgrid Research Activities, Symposium on microgrids, Montréal, June 2006

[10] C.P.Chang, C.W.Liu and C.C.Liu,"Unit commitment by Lagrangian relaxation and genetic algorithms", IEEE Trans Power Syst 15 (2000) (2), pp. 707-714.

[11]LI P., Formalisme de supervision des systèmes hybrides multi-sources de générateurs d'énergie répartie : Application aux micro-réseaux. Rapport de première année de thèse de l'Ecole Centrale de Lille, 2006

[12] HERBRETEAU J, COURTECUISSE V, LI P, DEGOBERT Ph,ROBYNS B, and FRANCOIS B." Association of PV, gas micro turbine and short term storage system to participate in frequency control”.

[13]BORNE P., ROZINOER J., DIEULOT J.Y., DUBOIS L., Introduction à la commande floue. Editions TECHNIP, Paris, 1998, 102p.

[14]ROBYNS B., FRANCOIS B., DEGOBERT P., HAUTIER J.P., Commande vectorielle de la machine asynchrone, Désensibilisation et optimisation par logique floue. Editions TECHNIP, Paris, 2007, 252p.

[15] Capstone Turbine Corporation, 410004 Rev. D (April 2006). Technical Reference.

[16] Ph. Degobert, S. Kreuawan, X. Guillaud, "Micro-grid powered by photovoltaic and micro turbine”, International Conference on Renewable Energy and Power Quality (ICREPQ'06) April 2006, Palma de Mallorca, Spain, CDROM.

[17] G. Boukettaya, L. Krichen, A. Ouali, "Fuzzy logic supervisor for power control of an isolated hybrid energy production unit”, International Journal of Electrical and Power Engineering” p 279-285, 2007 